\title{
Cloning and Characterization of a Novel Six-Transmembrane Protein STEAP2, Expressed in Normal and Malignant Prostate
}

\author{
Kati P. Porkka, Merja A. Helenius, and Tapio Visakorpi \\ Laboratory of Cancer Genetics, Institute of Medical Technology, University of Tampere, and Tampere University \\ Hospital, Tampere, Finland
}

\begin{abstract}
SUMMARY: By using subtraction and cDNA array hybridizations, we recently identified an anonymous transcript that was differentially expressed in benign prostate hyperplasia and prostate cancer cell line PC-3. Here, we report the cloning of the full-length cDNA of the gene, designated STEAP2 (six-transmembrane epithelial antigen of the prostate 2). The gene is located at the chromosomal region 7q21 and encodes for a 490-amino acid protein with six predicted transmembrane domains and is predominantly expressed in prostate epithelial cells. Green fluorescent protein fusion construct indicated that the STEAP2 protein is localized mainly in the plasma membrane. Real-time quantitative RT-PCR showed that the gene is expressed at levels more than 10 times higher in normal prostate than in other tissues studied. Of the prostate cancer cell lines, STEAP2 was expressed in significant levels only in androgen-responsive LNCaP. The expression of STEAP2 was significantly higher $(p=0.002)$ in both untreated primary and hormone-refractory prostate carcinomas than in benign prostate hyperplasias, suggesting that it may be involved in the development of prostate cancer. As a cell-surface antigen, STEAP2 is a potential diagnostic or therapeutic target in prostate cancer. (Lab Invest 2002, 82:1573-1582).
\end{abstract}

$$
T
$$
he identification of novel diagnostic markers as well as therapeutic targets is of utmost importance for improving the treatment of prostate cancer. One option for detecting novel cancer-associated genes is to identify genes that are differentially expressed in cancer cells. Various techniques for studying differential expression, such as differential display (Liang and Pardee, 1992), representational difference analysis (Hubank and Schatz, 1994), serial analysis of gene expression (Velculescu et al, 1995), suppression subtraction hybridization (Diatchenko et al, 1996), and cDNA microarray hybridization (Schena et al, 1995), have been developed. These analyses have already revealed several differentially expressed novel genes in prostate cancer, eg, PSCA, STEAP, TRPS1 (also known as GC79), and PCA3 (also known as DD3) (Bussemakers et al, 1999; Chang et al, 1997; Hubert et al, 1999; Reiter et al, 1998).

We recently combined suppression subtraction hybridization and cDNA array hybridization to detect genes that are differentially expressed in prostate

\section{DOI: 10.1097/01.LAB.0000038554.26102.C6}

Received August 28, 2002.

This study was supported by the Academy of Finland, the Cancer Society of Finland, the Reino Lahtikari Foundation, the Medical Research Fund of Tampere University Hospital, the Sigrid Juselius Foundation, the Cancer Society of Pirkanmaa, and the Finnish Life and Pension Insurance companies.

Address reprint requests to: Dr. Tapio Visakorpi, Institute of Medical Technology, FIN-33014 University of Tampere, Tampere, Finland. E-mail: tapio.visakorpi@uta.fi cancer (Porkka and Visakorpi, 2001). We first subtracted cDNA from benign prostate hyperplasia $(\mathrm{BPH})$ with cDNA from the PC-3 prostate cancer cell line. Next, the subtracted cDNA library was screened with the use of filter array technology. One of the identified differentially expressed genes was an anonymous expressed sequence tag (EST). Here, we describe the cloning and characterization of the gene, named STEAP2. The gene is expressed predominantly in the plasma membrane of prostate epithelial cells, and it encodes for a putative six-transmembrane protein with close homology to three other recently cloned six-transmembrane proteins. We also found that the expression of STEAP2 is significantly elevated in prostate cancer.

\section{Results}

\section{Cloning of STEAP2}

In BLAST analysis, the original 600-bp differentially expressed cDNA fragment (Porkka and Visakorpi, 2001) matched to the BAC clone RG016J04 (accession no. AA002064) from the chromosomal region $7 \mathrm{q} 21.13$ and to several anonymous ESTs (eg, Al023663, Al051210, R09227). Cloning of the fulllength CDNA (the sequence is deposited to the GenBank under accession no. AF455138) revealed four different transcripts, three of them resulting from differential splicing of the last exon. According to the Northern hybridizations, with three probes matching to the different splice variants, the longest version of the last exon, containing bases 24,610 to 29,888 of 
the sequence in the BAC clone RG016J04, turned out to be the major transcript. According to the Northern analysis, the length of the major transcript was approximately $7.5 \mathrm{~kb}$. The fourth splice variant resulted from a differentially spliced 113-bp exon at the $5^{\prime}$ untranslated region. The exon-intron organization of the gene and the putative open reading frames are illustrated in Figure 1.

\section{Sequence Variations}

Southern analysis did not show any major rearrangements of the gene in any of the prostate cancer cell lines studied (PC-3, DU145, and LNCaP). Also, sequencing of the exons did not reveal any nonsense or frameshift mutations in the cell lines PC-3, DU145LNCaP, and 22Rv1. However, four sites of base variations from the cell lines, as well as from a normal prostate cDNA pool, were discovered. One of the alterations was a neutral substitution at the nucleotide at codon 272 from CTC to CTT with no amino acid change, whereas the three others were missense substitutions: TाT $\rightarrow$ TGT leading to Phe17Cys, $\mathrm{CGA} \rightarrow \mathrm{CAA}$ leading to Arg456GIn, and ATG $\rightarrow$ ATT resulting in Met475lle. To study whether the detected alterations were mutations in the cell lines or polymorphic sites, we sequenced the four sites from 10 normal human DNA samples (20 chromosomes). All of the sites turned out to be polymorphic, with the allelic frequencies of $45 \%$ for $\mathrm{T}$ (Phe) and $55 \%$ for $\mathrm{G}$ (Cys) at codon 17, $45 \%$ for $\mathrm{C}$ and $55 \%$ for $\mathrm{T}$ (both coding for leucine) at codon $272,40 \%$ for $\mathrm{G}$ (Arg) and $60 \%$ for $\mathrm{A}$ (GIn) at codon 456, and 10\% for G (Met) and 90\% for $\mathrm{T}$ (Ile) at codon 475.

\section{Secondary Structure Prediction and Sequence Homology of the Protein}

The full-length cDNA contains a 1470-bp coding region of 490 amino acids with a predicted molecular mass of $56 \mathrm{kDa}$. According to PSORT II and SOSUI prediction programs, the protein is a membrane protein with six putative transmembrane domains (the membrane spanning regions predicted by PSORT II are the amino acids 216-232, 261-277, 307-323, $361-377,396-412$, and 433-449). The most likely localization of the protein was predicted to be either
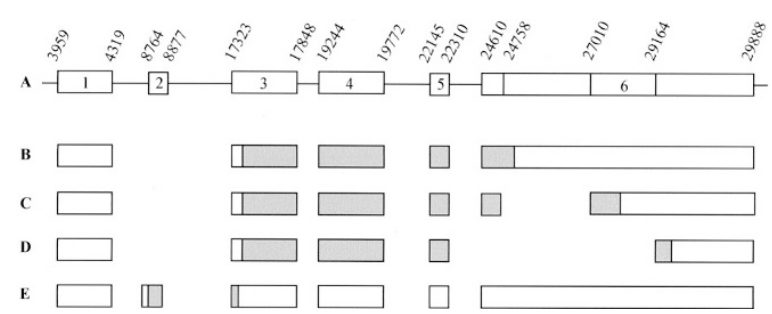

Figure 1.

A schematic presentation of the exon-intron organization of the STEAP2 gene. A, All exons that were discovered to be transcribed into mRNA in normal prostate. The base numbering refers to the genomic sequence in the BAC clone RG016J04 (accession no. AC002064). B, The major 7.5-kb transcript. C to E, The less abundant splicing forms of the gene. The putative coding regions of the variants are indicated in gray. endoplasmic reticulum (39.1\%) or plasma membrane (34.8\%). Nucleotide homology search with blastin did not reveal any significantly related genes. However, the analysis of amino acid homology with blastx algorithm showed three other six-transmembrane proteins with $50 \%$ identity and $70 \%$ similarity, on the average. These homologs were a human sixtransmembrane epithelial antigen of the prostate (STEAP) (Hubert et al, 1999) and its mouse counterpart (Yang et al, 2001), a rat tumor suppressor protein pHyde (Rinaldy and Steiner, 1999), and a tumor necrosis factor- $\alpha$-induced adipose-related protein (TIARP) (Moldes et al, 2001) from mouse. The rat pHyde and the mouse TIARP also have their human counterparts in the database (accession no. AAL78206, and NP_078912, respectively), but no other information about the human proteins is available. The alignment of the sequences is illustrated in Figure 2. During the submission process of this article, Korkmaz et al (2002) published the cloning of this same gene and named it six-transmembrane protein of prostate 1 (STAMP1). Its cDNA sequence (accession no. AY008445) is almost the same as described here with a slightly different 5 ' untranslated region. We named the novel gene STEAP2 because it shares significant similarities with human STEAP not only at the amino acid level but also at the expression pattern level, as described below.

\section{Subcellular Localization of the STEAP2 Protein}

The STEAP2 coding region fused with greenfluorescence protein (GFP) was expressed in LNCaP and DU145 cells to localize the STEAP2 protein. In both cell lines, the green fluorescent signal was visible in the plasma membrane and in the Golgi area, as well as in vesicle-like structures all over the cytoplasm. However, no signal appeared in the nucleus (Fig. 3A). The results were identical with the $\mathrm{N}$-terminal and the C-terminal fusion proteins. The cells that expressed only GFP without STEAP2 fusion showed the green fluorescence both in the cytoplasm and in the nucleus, as expected.

\section{Expression Analyses}

In the Northern analysis of normal tissues, STEAP2 was expressed predominantly in the prostate, showing some expression also in the ovary (Fig. 4A). Real-time RT-PCR assay for STEAP2 was set up to increase the sensitivity of the measurement of expression, as well as to quantify the relative expression (Fig. 5). In the real-time RT-PCR, the expression of STEAP2 was detected, in addition to the prostate, also in the skeletal muscle, mammary gland, testis, uterus, heart, kidney, lung, trachea, brain, and liver but at levels 10-fold lower than in the prostate (Fig. 4B). To study whether the expression of STEAP2 was of epithelial or stromal origin, we performed mRNA in situ hybridization. The results indicated that the epithelial and carcinoma cells are 


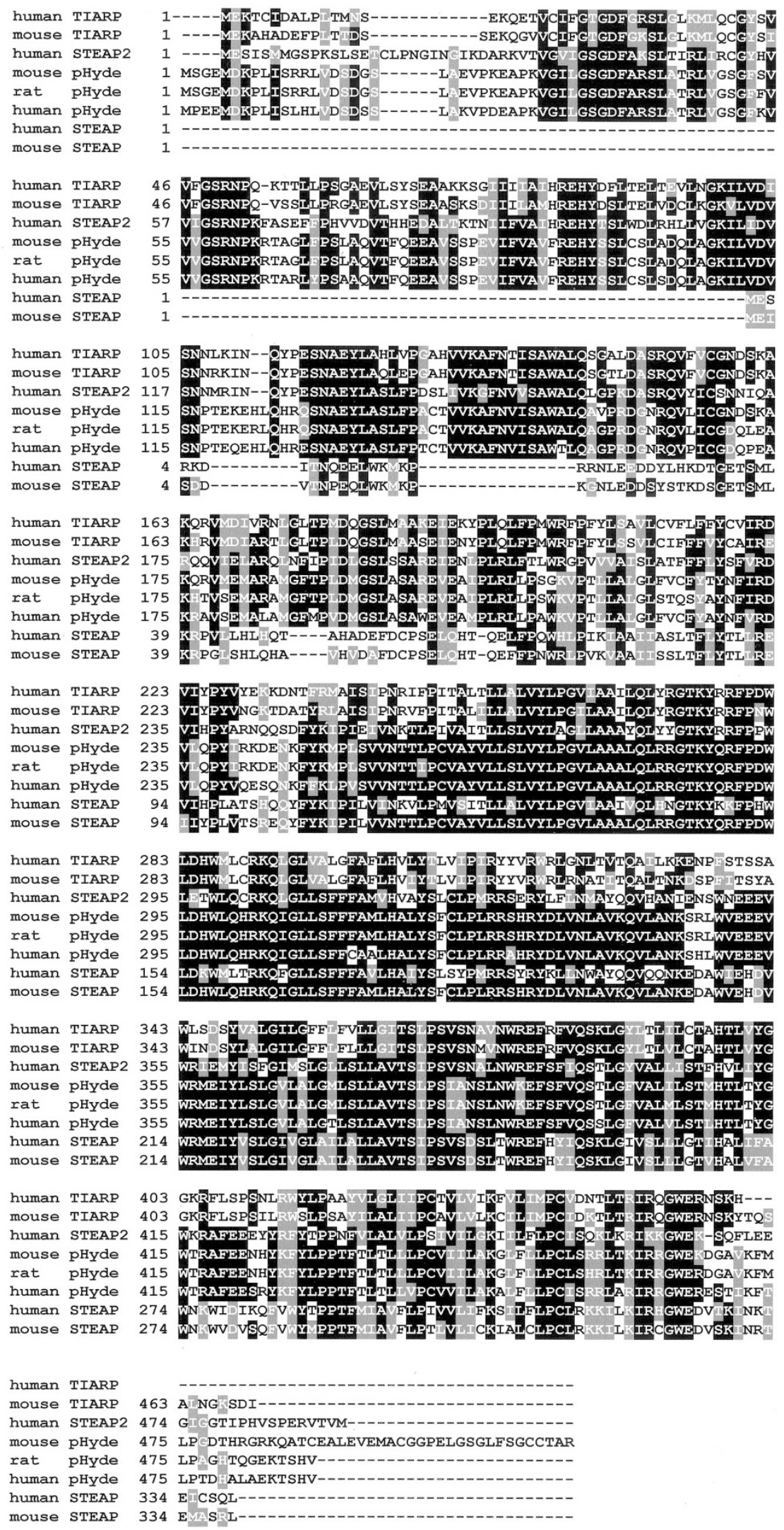

Figure 2.

Alignment of the homologous six-transmembrane proteins. The identical amino acids are shaded in black, and the similar amino acids are shaded in gray. The alignment was done by using CLUSTALW algorithm. The GenBank accession numbers of the protein sequences used for the alignment are NP_078912 (human homolog to TIARP), CAC41351 (mouse TIARP), AAK50539 (mouse homolog to pHyde), AAK00361 (rat pHyde), AAL78206 (human homolog to pHyde), AAF17479 (human STEAP), and AAK50537 (mouse homolog to STEAP). 

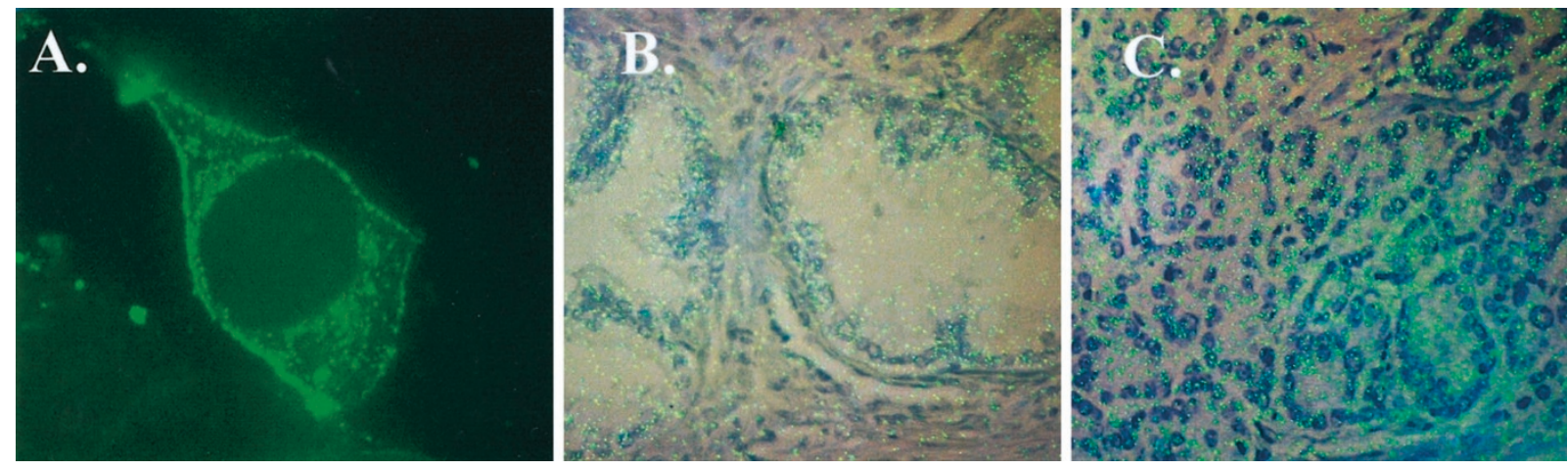

Figure 3.

Cellular expression of STEAP2. A, N-terminal GFP fusion protein of STEAP2 (green) is localized in the plasma membrane as well as in vesicle-like structures in the cytoplasm of DU145 cells. mRNA in situ hybridization with the STEAP2 antisense probe showing the expression of STEAP2 mRNA in the epithelial cells of benign prostate hyperplasia (B) and in carcinoma cells (C). Only a few silver grains are seen in the stroma, indicating that the epithelial cells are the major source of STEAP2 expression in prostate.
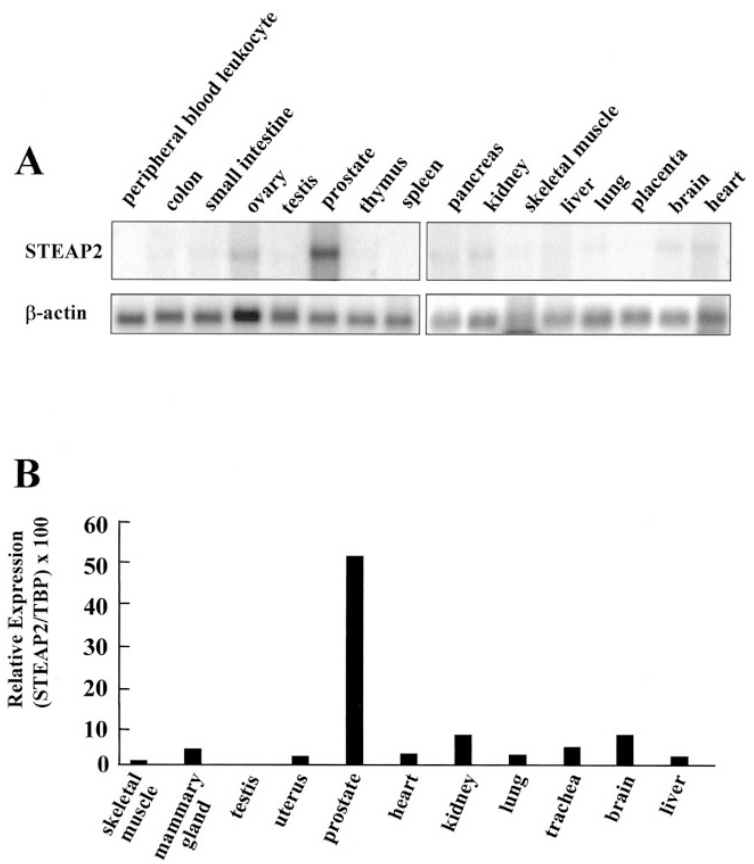

Figure 4.

Expression of STEAP2 in human tissues and in prostate cancer by Northern hybridization and by real-time quantitative RT-PCR. A, Northern hybridization showing the expression of STEAP2 in 16 human tissues. As a loading control, the same blot was hybridized with $\beta$-actin. B, Real-time quantitative RT-PCR showing the expression of STEAP2 in 11 human tissues.

the major source of STEAP2 expression in the prostate (Fig. 3B and $3 \mathrm{C}$ ).

Of the prostate cancer cell lines, only LNCaP showed strong expression, whereas in PC-3, DU145, and 22Rv1, the expression was either very weak or not detected by Northern blot. Quantitative RT-PCR showed that STEAP2 was expressed at levels approximately 30 -fold higher in LNCaP than in the other cell lines (Fig. 6A).

The expression of STEAP2 was measured by realtime quantitative RT-PCR also in BPH, in untreated as well as in hormone-refractory prostate carcinomas. STEAP2 was expressed, on the average, at levels 3 -fold higher in carcinomas than in BPH $(p=0.002)$.
The level of expression in untreated and hormonerefractory carcinomas was approximately the same (Fig. 6B). There was no significant association between the histological grade (World Health Organization grading system) and the level of STEAP2 expression in untreated primary tumors.

Of all the cell lines studied here, LNCaP is the only androgen-dependent cell line. To test whether the expression of STEAP2 is regulated by androgens, we cultured LNCaP cells in the presence of different concentrations of dihydrotestosterone (DHT) and in the absence of androgens. No differences in the expression of STEAP2 between androgen-deprived and DHT-stimulated LNCaP cells were detected in Northern analysis, whereas the expression of prostate specific antigen, a known androgen-regulated gene, correlated with the concentration of DHT (Fig. 7).

One possible mechanism for the down-regulation of the STEAP2 gene in the androgen-independent cell lines is hypermethylation of the promoter region of the gene. To test this possibility, we cultured PC-3 and DU145 cells in the presence of a demethylating agent, 5-aza-2'-deoxycytidine. The treatment, however, did not restore the expression of STEAP2. As a positive control of the demethylation treatment, the expression of estrogen receptor- $\alpha$, known to be hypermethylated in DU145, was also analyzed. As expected, the expression of estrogen receptor- $\alpha$ was restored by the demethylation (Fig. 8).

\section{Discussion}

By combining cDNA subtraction and array hybridization, we recently identified an anonymous EST that was expressed in BPH but not in the PC-3 prostate cancer cell line. Here, we have cloned and characterized this novel gene named STEAP2. The gene is located at the chromosomal region $7 \mathrm{q} 21$, and it encodes for 490 amino acid protein. According to secondary structure predictions, STEAP2 protein contains six transmembrane domains and is localized in either the endoplasmic reticulum or the plasma membrane. The analysis of GFP fusion protein verified that 

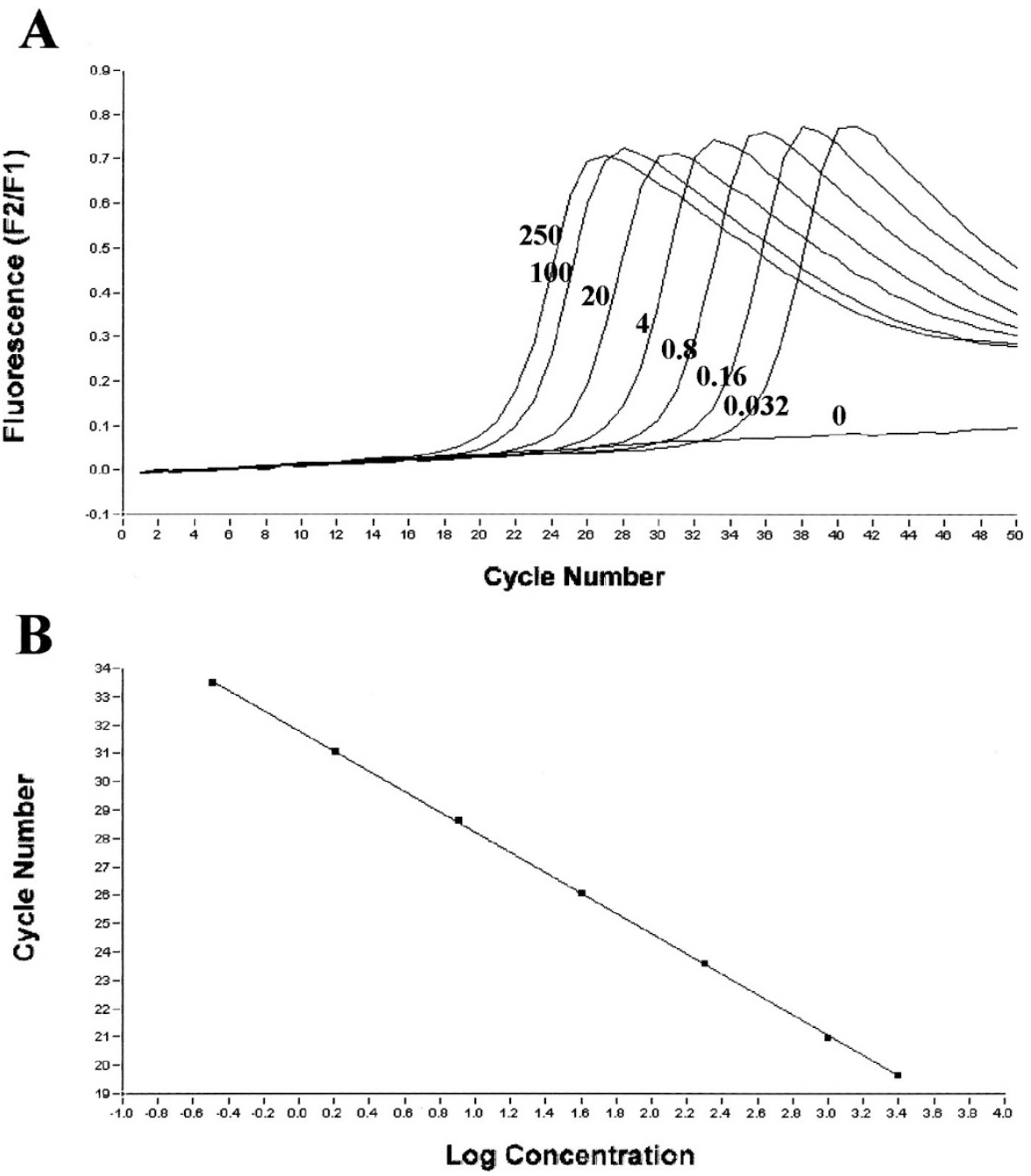

Figure 5.

Real-time RT-PCR assay for STEAP2. A, PCR curves of the serially diluted standard containing CDNA transcribed from 250, 100, 20, 4, 0.8, 0.16 , and $0.032 \mathrm{ng}$ of total RNA. B, Standard curve blotting fractional cycle number at the fluorescent threshold against the logarithmic concentration of each standard.

STEAP2 is localized mostly in the plasma membrane and Golgi area. In normal tissues, STEAP2 was predominantly expressed in the prostate. In addition, significant levels of expression were found only in the ovary. The expression in other tissues was so low that STEAP2 is unlikely to possess any functional significance in these tissues. mRNA in situ hybridization indicated that the gene is expressed mostly in the epithelial cells of the prostate. During the submission process of this article, the same gene was cloned and published by Korkmaz et al (2002) under the name STAMP1. Our findings are generally in good agreement with the data published by Korkmaz et al (2002). Some minor differences in the findings are that Korkmaz et al (2002) reported that in Northern analysis, they found also minor bands of $2.2,4.0$, and $4.5 \mathrm{~kb}$ in size in prostate tissue. We did not see those bands, although the cloning process did reveal several splice variants (Fig. 1). In addition, Korkmaz et al (2002) estimated, on the basis of Northern analysis, that the size of the major transcript is $6.5 \mathrm{~kb}$, whereas we estimated it to be $7.5 \mathrm{~kb}$.
Of the five prostate cancer cell lines studied, STEAP2 was expressed only in androgenresponsive LNCaP. However, stimulation of the LNCaP cells with DHT did not increase the expression of STEAP2. In addition, Korkmaz et al (2002) showed that the expression of the gene is not altered in vivo in nude mice upon castration. Thus, it seems that androgens do not directly induce the expression of STEAP2. No mutations in the coding region of the gene, major genomic rearrangements, or promoter hypermethylation were found in the cell lines, either. Thus, the exact mechanisms of the loss of expression of STEAP2 in the androgenindependent cell lines, including PC-3, remain unknown. However, the androgen-independent cell lines are known for the loss of expression of many genes, such as the androgen receptor, that are commonly expressed in both untreated and hormone-refractory prostate carcinomas in vivo (Linja et al, 2001). Therefore, it is important also to analyze prostate tumors, in addition to the cell lines. 
A.

B.
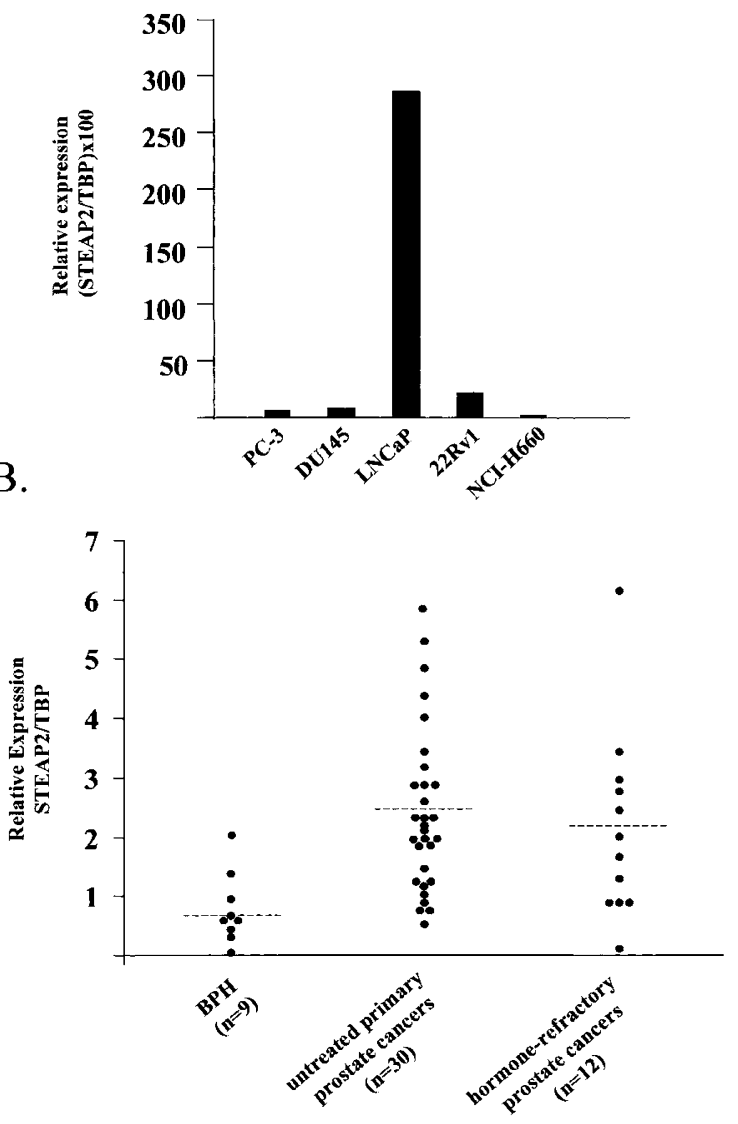

Figure 6 .

Expression of STEAP2 in prostate cancer cell lines and tumors by real-time quantitative RT-PCR. A, LNCaP showed approximately 30-fold higher expression of STEAP2 than the other cell lines. B, Prostate cancers express significantly more STEAP2 than benign prostate hyperplasia $(p=0.002$, Kruskal-Wallis test). Dotted lines indicate mean level of expression.

Consequently, we measured the expression of STEAP2 in prostate cancer specimens using real-time quantitative RT-PCR. The analyses showed that STEAP2 was expressed at significantly higher levels in prostate cancer than in $\mathrm{BPH}$. By using nonquantitative mRNA in situ hybridization also, Korkmaz et al (2002) showed that the expression of STAMP1 (also known as STEAP2) is increased in prostate cancer. Here, however, no differences were found between untreated and hormone-refractory prostate carcinomas. The STEAP2 gene is located in chromosome 7q, which is one of the most commonly gained chromosome arms in prostate cancer (Visakorpi et al, 1995). The gain of chromosome 7 has also been associated with the aggressive behavior of prostate cancer (Alcaraz et al, 1994). Whether the high expression of STEAP2 is associated with the gain of chromosome 7 remains to be studied. It is interesting that three missense polymorphisms were found in the gene. Whether any of them have any functional significance and whether any of the alleles are associated with the risk of prostate cancer also remain to be studied.

The various prediction programs did not reveal any functional domains for the STEAP2 protein. Thus, the
DHT (M)

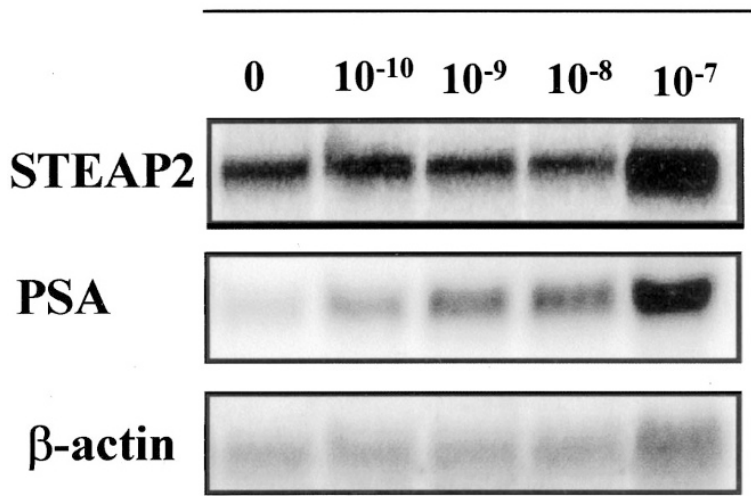

Figure 7.

Androgen regulation of STEAP2 expression. Northern hybridization showing the expression of STEAP2 and prostate-specific antigen (PSA) in LNCaP cells cultured in the absence of dihydrotestosterone (DHT; $0 \mathrm{M}$ ) as well as with increasing concentrations of DHT $\left(10^{-10}-10^{-7} \mathrm{M}\right)$. No differences in the expression of STEAP2 are detected, whereas the expression of PSA increases with the concentration of DHT, as expected. As a loading control, the same blot was hybridized with $\beta$-actin. Slightly stronger hybridization signal of STEAP2 in the last lane was due to overloading of the last RNA sample.

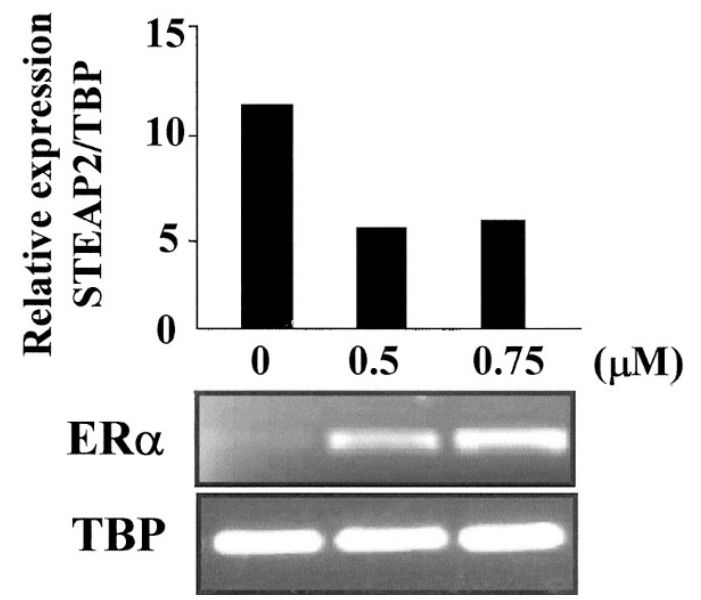

Figure 8.

Hypermethylation of STEAP2. RT-PCR analysis shows the expression of STEAP2 in DU145 cells after the treatment with $0-, 0.5-$, or $0.75-\mu \mathrm{m}$ concentration of a demethylating agent 5-aza-2'-deoxycytidine. The treatment did not alter the expression of STEAP2, whereas the expression of estrogen receptor- $\alpha$ was restored by 5 -aza-2'-deoxycytidine, as expected. TATA-box binding protein was used for normalization.

putative function of the gene can be foreseen only on the basis of the similarity of the protein with the other proteins. Recently, three other six-transmembrane proteins, closely resembling the STEAP2 protein, have been cloned and characterized: a human sixtransmembrane epithelial antigen of the prostate (STEAP) (Hubert et al, 1999), a rat tumor suppressor pHyde (Rinaldy and Steiner, 1999), and a mouse tumor necrosis factor- $\alpha$-induced adipose-related protein (TIARP) (Moldes et al, 2001). All of them, together with the STEAP2 protein, share a significant homology at the amino acid level with $50 \%$ identity and $70 \%$ similarity, on the average, indicating that they belong 
to the same gene family. It is interesting that STEAP, STEAP2, and human TIARP are all located at the chromosomal region $7 q 21$. Similarly to STEAP2, the expression of STEAP has been reported to be prostate-specific and higher in LNCaP than in DU145 or PC-3 (Hubert et al, 1999).

Human STEAP was originally cloned from a prostate cancer xenograft model mimicking an advanced metastatic disease. The STEAP protein is located mostly at the cell-cell boundaries, suggesting that it functions as a channel in cell junctions (Hubert et al, 1999). As with the human STEAP, the mouse TIARP protein is reported to be located mainly at the cell-cell junctions, as well as in plasma membrane invaginations. The expression of TIARP is induced by tumor necrosis factor- $\alpha$ in differentiating and in mature mouse 3T3-L1 cells. TIARP is also expressed spontaneously during the differentiation of adipocytes (Moldes et al, 2001). The gene coding for the rat protein pHyde was first cloned from a high-metastatic (MAT-LyLu) Dunning R3227 rat prostatic cell line (Rinaldy and Steiner, 1999). In additional studies $p H y d e$ has been shown to operate as an intrinsic up-regulating factor for the apoptotic pathway in prostate cancer (Rinaldy et al, 2000). Overexpression of pHyde significantly inhibited the growth of human prostate cancer cell lines DU145 and LNCaP in culture, and injection of pHyde-expressing construct reduced the growth of DU145 tumors in nude mice. Therefore, the authors suggested that $p H y d e$ is a novel tumor suppressor gene (Steiner et al, 2000). In contrast, the finding that STEAP2 was overexpressed in prostate cancer suggests that STEAP2 may actually possess oncogenic properties. Obviously, functional studies are needed to elucidate the possible involvement of STEAP2 in cell proliferation, apoptosis, and tumor growth.

In conclusion, we have cloned and characterized a novel gene encoding a prostate-specific transmembrane protein. The high expression of the gene in prostate cancer suggests that the gene may be involved in the development of prostate cancer. The plasma membrane localization of the protein warrants the studying of the possible diagnostic utility of the protein, either as an early detection marker or as a target for imaging. In addition, as a cell-surface antigen, STEAP2 is a potential candidate for the immunotherapy of prostate cancer. The relatively prostatespecific nature of the gene's expression suggests that the promoter of the gene may also become useful in targeting expression of, for example, therapeutic genes to prostate epithelial cells.

\section{Materials and Methods}

\section{Cell Lines and Tissue Material}

The prostate cancer cell lines PC-3, DU145, LNCaP, $\mathrm{NCl}-\mathrm{H} 660$, and 22Rv1 were obtained from the American Type Culture Collection (Rockville, Maryland) and cultured under the recommended condi- tions. Total RNA from the cell lines was extracted using TRIzol reagent (Life Technologies, Grand Island, New York). Commercially available Human Prostate Poly $\mathrm{A}^{+}$and total RNAs from normal human tissues (Clontech Laboratories Inc., Palo Alto, California) were used for cloning the full-length cDNA and for RT-PCR experiments, respectively. Genomic DNA was extracted from the cell lines using standard techniques (Sambrook et al, 1989). In addition, DNA from 10 healthy blood donors was obtained for analysis of polymorphism. For the analysis of STEAP2 expression by RT-PCR, freshly frozen specimens of $9 \mathrm{BPH}, 30$ primary prostate carcinomas (prostatectomy specimens), and 12 locally recurrent hormone-refractory prostate carcinomas (transurethral resection specimens from patients under hormonal therapy) were obtained from the Tampere University Hospital. The specimens were first histologically examined for the presence of $>60 \%$ of carcinoma or hyperplastic tissue using hematoxylin and eosin-stained slides. The BPH samples were obtained from prostatectomy specimens from cancer patients. However, samples were checked carefully to ensure that they did not contain carcinoma cells and that they consisted of mostly hyperplastic epithelial cells. Next, a small amount of tissue was scraped just beneath the section used for histological examination and used for total RNA isolation by using the Qiagen RNeasy MiniKit (Qiagen Inc., Valencia, California). For the mRNA in situ hybridization analyses, paraffin-embedded samples of $\mathrm{BPH}$ and carcinoma were obtained from the Tampere University Hospital (Tampere, Finland).

\section{Cloning of the Full-Length cDNA}

To clone the full-length cDNA of STEAP2, we first used virtual cloning. cDNA contig was built from EST sequences using publicly available databases. The genomic sequence of STEAP2 (accession no. AC002064) was used to make exon predictions using Grail (grail.Isd.ornl.gov/grailexp/) and Genscan (genes.mit.edu/GENSCAN.html) programs. The final $5^{\prime}$ and $3^{\prime}$ ends of the STEAP2 cDNA were cloned using the SMART RACE protocol according to the manufacturer's instructions (Clontech Laboratories Inc.). Sequencing was performed using an ABI PRISM BigDye Terminator Cycle Sequencing Ready Reaction Kit and an $\mathrm{ABI} 310$ sequencer (PerkinElmer Life Science Inc., Boston, Massachusetts). Sequence similarity search was carried out using the BLAST with the blastn and blastx algorithms (www.ncbi.nlm.nih.gov/BLAST/). PSORT ॥ (psort.nibb.ac.jp/) and SOSUI (sosui.proteome.bio.tuat.ac.jp/sosuiframe0.html) programs were used to predict the secondary structure and subcellular localization of the putative protein.

\section{Southern Hybridization}

For screening the STEAP2 gene for rearrangements, $30 \mu \mathrm{g}$ of genomic DNA from the cell lines PC-3, 
DU145, and LNCaP and from normal human DNA was digested with $\mathrm{Xbal}$ and separated in $0.8 \%$ agarose gel electrophoresis. The gel was then blotted using routine techniques (Sambrook et al, 1989). The full-length STEAP2 cDNA was labeled with ${ }^{32} \mathrm{P}$-dCTP using the Rediprime II random prime labeling system (Amersham Pharmacia Biotech, Uppsala, Sweden). The overnight hybridization and washes were carried out as previously described (Sambrook et al, 1989). The blot was visualized with the Storm Phosphoimager (Molecular Dynamics Inc., Sunnyvale, California).

\section{Northern Hybridization}

Twenty micrograms of total RNA was electrophoresed in agarose-formaldehyde gel, blotted, and hybridized using standard protocols (Sambrook et al, 1989). In addition, commercial MTN blots (Clontech Laboratories Inc.) were hybridized according to the manufacturer's instructions. The cDNA clones for STEAP2 and for prostate-specific antigen probes were obtained from the subtracted library (Porkka and Visakorpi, 2001). The probes were labeled with ${ }^{32}$ P-dCTP using the Rediprime II random prime labeling system (Amersham Pharmacia Biotech). The blots were visualized with Storm Phosphoimager (Molecular Dynamics Inc.). Visualization of $18 \mathrm{~S}$ and 28S RNAs, as well as hybridization with probe for $\beta$-actin, was used to control equal loading of the gels.

\section{Real-Time Quantitative RT-PCR}

The real-time quantitative RT-PCR was performed according to previously published guidelines (Linja et al, 2001). Briefly, first-strand cDNA was synthesized from total RNA with Superscript II reverse transcriptase (Life Technologies) and oligo $d(T)_{12-18}$ primer. For preparing the standard curve, $5 \mu \mathrm{g}$ of total RNA from normal prostate tissue (Clontech Laboratories Inc.) was reverse-transcribed, after which serial dilutions were made corresponding to cDNA transcribed from $250,100,20,4,0.8,0.16$, and $0.032 \mathrm{ng}$ of total RNA. The primer sequences used for the analysis of STEAP2 expression were 5'-TGGAATGAGGAAGAAGTITGGA3'and 5'-GCAAGAACAAAGTTTGGTGGTGTA-3', and for the probe sequences 5'-TGGCTTACTITCCCTCCTGGCAGT-3'-fluorescein, as well as Red640-5'-CTTCTATCCCTTCAGTGAGCAATGCTाT-3' (purchased from TibMolBiol, Berlin, Germany). The PCR reactions were performed in the LightCycler apparatus using the LC FastStart DNA Hybridization Probes Kit according to the manufacturer's instructions (Roche Diagnostics, Mannheim, Germany). After 10 minutes of initial denaturation at $95^{\circ} \mathrm{C}$, the cycling conditions (55 cycles) were as follows: denaturation at $95^{\circ} \mathrm{C}$ for 10 seconds, annealing at $58^{\circ} \mathrm{C}$ for 13 seconds, and elongation at $72^{\circ} \mathrm{C}$ for 10 seconds. The fluorescence of each sample was measured in every cycle at the end of the annealing step. After proportional background adjustment, the fit point method was used to determine the cycle in which the log-linear signal is distinguished from the background, and that cycle number was used as a crossing-point value. Software produced the standard curve by measuring the crossing point of each standard and plotting them against the logarithmic value of concentrations. The concentrations of unknown samples were then calculated by setting their crossing points to the standard curve. The intraassay coefficient of variation, determined by repeating analyses of two samples 10 times, was 6.5\%. For normalization of the expression levels, the expression of TATA-box binding protein (TBP) was measured as previously described (Linja et al, 2001). The relative expression of STEAP2 was calculated by dividing the STEAP2 value by the TBP value of each sample.

\section{Androgen Stimulation of the LNCaP Cells}

The androgen receptor-positive LNCaP cells were cultured in phenol-red free RPMI medium (Sigma Chemical Co., St. Louis, Missouri) with 10\% charcoal-stripped serum (HyClone Laboratories Inc., Logan, Utah) for 24 hours before androgen stimulation. The medium was then replaced with fresh phenol-red free RPMI medium containing 10\% charcoal-stripped serum and dihydrotestosterone (DHT, D5027; Sigma Chemical Co.) at final concentrations of $0 \mathrm{M}, 10^{-10} \mathrm{M}, 10^{-9} \mathrm{M}, 10^{-8} \mathrm{M}$, and $10^{-7} \mathrm{M}$. The cells were cultured with DHT for 24 hours and then harvested for RNA extraction. Total RNA was extracted from the cells using an RNeasy Mini Kit (Qiagen GmbH, Hilden, Germany) and used for Northern hybridization.

\section{Methylation Analysis}

The prostate cancer cell lines PC-3 and DU145, showing no expression of STEAP2, were cultured in RPMI with $10 \%$ fetal bovine serum to $50 \%$ confluence. Next, the medium was replaced with fresh medium containing the demethylating agent 5-aza-2'-deoxycytidine (A3656; Sigma Chemical Co.) at final concentrations of $0,0.5$, and $0.75 \mu \mathrm{M}$. The cells were redosed after every 48 hours and harvested after 5 days. Total RNA was extracted using TRIzol-reagent (Life Technologies), and the expression of STEAP2 was studied by using both Northern hybridization and quantitative RT-PCR as described above. As a positive control, the expression of estrogen receptor- $\alpha$, a gene that has been shown to be hypermethylated in DU145, was studied by RT-PCR as described (Li et al, 2000).

\section{Subcellular Localization of STEAP2 with GFP Fusion Proteins}

The coding region of STEAP2 was PCR-amplified using gene-specific primers with $\mathrm{Kpnl}$ and Bg/ll restriction enzyme recognition sites. After digestion, the PCR products were cloned into pEGFP-N3 and pEGFP-C1 vectors (Clontech Laboratories Inc.) to produce N-terminal and C-terminal GFP fusion constructs. LNCaP and DU145 cells were grown to $80 \%$ confluence and transfected with 6 to $8 \mu \mathrm{g}$ of the 
purified plasmids using the lipofection method (Lipofectamine PLUS reagent; Life Technologies). Two days after the transfection, the cells were fixed with $4 \%$ paraformaldehyde/5\% saccharose/PBS for 30 minutes, counterstained with an antifade solution (Vectashield; Vector Laboratories, Burlingame, California) containing 4,6-diamidino 2-phenylindole, and visualized using confocal laser scanning microscopy (UltraView; PerkinEImer Life Science Inc.).

\section{In Situ Hybridization}

For the mRNA in situ hybridization, the original 600-bp cDNA fragment from the subtraction library (Porkka and Visakorpi, 2001) was subcloned into a pBluescript vector, which was subsequently linearized to generate STEAP2 antisense and sense probes by in vitro transcription. Formalin-fixed, paraffin-embedded prostate tissue sections were hybridized using ${ }^{33} \mathrm{P}$-labeled, in vitro-transcribed probes as previously described (Nupponen et al, 1999). The sections were counterstained with hematoxylin and imaged using a Nikon Microphot-SA fluorescence microscope (Nikon Corporation, Tokyo, Japan) with an epipolarization filter allowing simultaneous visualization of grains and tissue morphology.

\section{Statistical Analysis}

The association of the gene expression levels with tumor type and histological grade was calculated with a nonparametric Kruskal-Wallis test and a Dunn's multiple comparisons test.

\section{Acknowledgements}

We are grateful to Ms. Mariitta Vakkuri, Ms. Heli Lehtonen, and Ms. Maarit Ohranen for technical assistance.

\section{References}

Alcaraz A, Takahashi S, Brown JA, Herath JF, Bergstralh EJ, Larson-Keller JJ, Lieber MM, and Jenkins RB (1994). Aneuploidy and aneusomy of chromosome 7 detected by fluorescence in situ hybridization are markers of poor prognosis in prostate cancer. Cancer Res 54:3998-4002.

Bussemakers MJ, van Bokhoven A, Verhaegh GW, Smit FP, Karthaus HF, Schalken JA, Debruyne FM, Ru N, and Isaacs WB (1999). DD3: A new prostate-specific gene, highly overexpressed in prostate cancer. Cancer Res 59:5975-5979.

Chang GT, Blok LJ, Steenbeek M, Veldscholte J, van Weerden WM, van Steenbrugge GJ, and Brinkmann AO (1997). Differentially expressed genes in androgen-dependent and -independent prostate carcinomas. Cancer Res 57:40754081.

Diatchenko L, Lau YF, Campbell AP, Chenchik A, Moqadam F, Huang B, Lukyanov S, Lukyanov K, Gurskaya N, Sverdlov ED, and Siebert PD (1996). Suppression subtractive hybridization: A method for generating differentially regulated or tissue-specific cDNA probes and libraries. Proc Natl Acad Sci USA 93:6025-6030.
Hubank M and Schatz DG (1994). Identifying differences in mRNA expression by representational difference analysis of cDNA. Nucleic Acids Res 22:5640-5648.

Hubert RS, Vivanco I, Chen E, Rastegar S, Leong K, Mitchell SC, Madraswala R, Zhou Y, Kuo J, Raitano AB, Jakobovits A, Saffran DC, and Afar DE (1999). STEAP: A prostate-specific cell-surface antigen highly expressed in human prostate tumors. Proc Natl Acad Sci USA 96:14523-14528.

Korkmaz KS, Elbi CC, Korkmaz CG, Loda M, Hager GL, and Saatcioglu $F$ (2002). Molecular cloning and characterization of STAMP1, a highly prostate specific six-trans-membrane protein that is overexpressed in prostate cancer. J Biol Chem 277:36689-36696.

Li LC, Chui R, Nakajima K, Oh BR, Au HC, and Dahiya R (2000). Frequent methylation of estrogen receptor in prostate cancer: Correlation with tumor progression. Cancer Res 60:702-706.

Liang P and Pardee AB (1992). Differential display of eukaryotic messenger RNA by means of the polymerase chain reaction. Science 257:967-971.

Linja MJ, Savinainen KJ, Saramäki OR, Tammela TL, Vessella $\mathrm{RL}$, and Visakorpi T (2001). Amplification and overexpression of androgen receptor gene in hormone-refractory prostate cancer. Cancer Res 61:3550-3555.

Moldes M, Lasnier F, Gauthereau X, Klein C, Pairault J, Feve B, and Chambaut-Guerin AM (2001). Tumor necrosis factor-alpha-induced adipose-related protein (tiarp), a cell-surface protein that is highly induced by tumor necrosis factor-alpha and adipose conversion. $\mathrm{J}$ Biol Chem 276:33938-33946.

Nupponen NN, Porkka K, Kakkola L, Tanner M, Persson K, Borg Å, Isola J, and Visakorpi T (1999). Amplification and overexpression of p40 subunit of eukaryotic translation initiation factor 3 in breast and prostate cancer. Am J Pathol 154:1777-1783.

Porkka KP and Visakorpi T (2001). Detection of differentially expressed genes in prostate cancer by combining suppression subtractive hybridization and cDNA library array. J Pathol 193:73-79.

Reiter RE, Gu Z, Watabe T, Thomas G, Szigeti K, Davis E, Wahl M, Nisitani S, Yamashiro J, Le Beau MM, Loda M, and Witte ON (1998). Prostate stem cell antigen: A cell surface marker overexpressed in prostate cancer. Proc Natl Acad Sci USA 95:1735-1740.

Rinaldy AR, Menon RP, Lerner JL, and Steiner MS (2000). Role of pHyde novel gene product as an intrinsic factor for apoptotic pathway in prostate cancer. Gan To Kagaku Ryoho 27(Suppl 2):215-222.

Rinaldy AR and Steiner MS (1999). Application of an improved cDNA competition technique to identify prostate cancer-associated gene. DNA Cell Biol 18:829-836.

Sambrook J, Fritsch EF, and Maniatis T (1989). Molecular Cloning: A Laboratory Manual. Cold Spring Harbor, NY: Cold Spring Harbor Laboratory.

Schena M, Shalon D, Davis RW, and Brown PO (1995). Quantitative monitoring of gene expression patterns with a complementary DNA microarray. Science 270:467-470.

Steiner MS, Zhang X, Wang Y, and Lu Y (2000). Growth inhibition of prostate cancer by an adenovirus expressing a novel tumor suppressor gene, pHyde. Cancer Res 60:44194425 . 
Velculescu VE, Zhang L, Vogelstain B, and Kinzler KW (1995). Serial analysis of gene expression. Science 270: 484-487.

Visakorpi T, Kallioniemi A, Syvänen A-C, Hyytinen E, Karhu R, Tammela T, Isola J, and Kallioniemi O-P (1995). Genetic changes in primary and recurrent prostate cancer by comparative genomic hybridization. Cancer Res 55:342347.
Yang D, Holt GE, Velders MP, Kwon ED, and Kast WM (2001). Murine six-transmembrane epithelial antigen of the prostate, prostate stem cell antigen, and prostate-specific membrane antigen: Prostate-specific cell-surface antigens highly expressed in prostate cancer of transgenic adenocarcinoma mouse prostate mice. Cancer Res 61:58575860 . 